\section{Etiquetado y rotulación de los alimentos en la prevención del sobrepeso y la obesidad: una revisión sistemática}

\author{
Food labeling and the prevention of overweight \\ and obesity: a systematic review
}

\footnotetext{
1 Universidad de Alicante,

Alicante, España.

2 Universidad Miguel

Hernández, Elche, España.

3 Universidad Cardenal

Herrera, Valencia, España.

Correspondencia

J. Sanz-Valero

Universidad de Alicante. Campus de Sant Vicent del Raspeig. Apdo. Correos 99, Alicante/Alicante - 03080, España.

javier.sanz@ua.es
}

\begin{abstract}
This article reports on a systematic review of articles on food labeling and the prevention of obesity and overweight, in the MEDLINE, EMBASE, Web of Knowledge, Cochrane Library Plus, Food Science and Technology Abstracts, LILACS, and CINAHL databases. The DeCS/MeSH descriptors were obesity and food labeling. 207 articles were retrieved. Using inclusion and exclusion criteria, 14 articles were selected: 11 were on food labeling and its impact on final food product consumption; 2 were on fast food establishments; 1 on sensory attributes as compared to health recommendations; and 2 on follow-up of interventions. Labeling has a positive effect on final food product consumption, in contrast with fast food restaurants. Sensory attributes were more effective than recommendations on the labels. Follow-up of interventions confirmed the long-term effect of the target interventions.
\end{abstract}

Food Labeling; Obesity; Nutrition Policy; Food Habits; Feeding Behavior
Miren Itxaso Sebastián-Ponce 1

Javier Sanz-Valero 1,2

Carmina Wanden-Berghe 3

\section{Introducción}

Mientras en el tercer mundo muere una persona de hambre cada cuatro segundos, el mundo desarrollado se enfrenta a la epidemia del siglo XXI: la obesidad. Sólo en Europa, el $50 \%$ de los adultos son obesos o tienen sobrepeso. En los últimos 20 años, el número de ellos se ha triplicado y desde 1970 el número de niños con obesidad o sobrepeso se ha multiplicado por 10 1. Estos datos son similares en todos los países desarrollados.

La obesidad está asociada con problemas en la salud (diabetes, enfermedades cardiovasculares, cáncer, etc.). Esta situación implica un coste económico para los estados: sobre 120 billones de euros se invierten anualmente en enfermedades relacionadas con la obesidad 2. Es por esto, por la carga sanitaria y económica que implica la obesidad, que su prevención entra dentro del debate político de los países que la sufren.

La obesidad representa el triunfo de los alimentos energéticos y baratos, de las porciones grandes, de la omnipresencia de comida en cualquier punto de venta (desde las gasolineras hasta las escuelas de primaria) y de la incesante publicidad de comida de baja calidad nutricional y con un exceso de grasa. Además, los hábitos de las sociedades desarrolladas han cambiado y el ocio suele ser pasivo (internet, televisión, videojuegos) 3 . En esta línea, la tendencia actual es aumentar el consumo de comida rápida o para llevar, que puede llegar a suponer hasta un 
tercio de las calorías consumidas; y este tipo de comidas contiene más calorías, grasas saturadas y colesterol, y menos fibra, que las comidas caseras 4 . Además, las evidencias dicen que en este tipo de comidas la capacidad del individuo para conocer la composición de lo que ingiere es limitada, debido a la información parcial que se ofrece y a la escasa relación con lo que se consume 5 .

Las intervenciones que se proponen para la reducción de la obesidad pasan por la educación nutricional, la restricción de anuncios de comida basura en televisión, sobre todo los dirigidos a los niños (o al menos tener en cuenta el horario en el que se emiten), el fomento de los alimentos saludables, la reformulación de productos dentro de la industria alimentaria, las políticas nutricionales que graven más los productos con escaso valor nutritivo, la promoción de la actividad física, etc. 6,7 .

La educación nutricional tiene, entre otras tareas, la de enseñar a elegir alimentos saludables. El medio con el que se cuenta para informar sobre la composición nutricional de un alimento, es su etiqueta. La información en una etiqueta es esencial para hacer elecciones correctas. El etiquetado de los productos, de todos los productos, también los que se ofrecen en cáterin y comida para llevar, debe ser entendible por los consumidores ${ }^{8}$. El etiquetado es una forma directa, barata y factible de influir en la elección de los productos y puede actuar de forma muy positiva controlando el problema actual de la obesidad 9 . La comprensión de la etiqueta se apoya en ocasiones con logotipos que simplifican la elección del producto saludable 10, como por ejemplo: Pick the Tick, Traffic Light o Keyhole 4,11,12.

En consecuencia, y teniendo en consideración lo anteriormente expuesto, el objetivo de este trabajo es conocer cómo se ha actuado mediante el etiquetado o la rotulación en la prevención de la obesidad y el sobrepeso.

\section{Material y método}

\section{Diseño}

Estudio descriptivo transversal de los trabajos recuperados en la revisión bibliográfica mediante técnica sistemática.

\section{Fuente de obtención de los datos}

Todos los datos que se utilizaron en este estudio se obtuvieron de la consulta directa y acceso, vía Internet, a la literatura científica recogida en las siguientes bases de datos:
- Medlars Online International Literature (MEDLINE), via PubMed;

- EMBASE;

- Web of Knowledge, Institute for Scientific Information (ISI);

- The Cochrane Library Plus;

- Food Science and Technology Abstracts (FSTA);

- Latin American and Caribbean Heath Sciences Literature (LILACS);

- The Cumulative Index to Nursing and Allied Health Literature (CINAHL).

\section{Tratamiento de la información}

Se estudiaron los artículos publicados en cualquier país, por cualquier institución o investigador individual y en cualquiera de los idiomas, publicados desde el inicio de la indexación de cada una de las fuentes primarias.

Para la recuperación documental se emplearon los Descriptores en Ciencias de la Salud (DeCS) creados por OPS/BIREME a partir de los Medical Subject Headings (MeSH) desarrollados por la U.S. National Library of Medicine. No se utilizaron Calificadores de Materia (Subheadings), ni fue necesario en empleo de Tags.

Del estudio del Thesaurus se consideraron adecuados los descriptores "obesidad" (obesity) y "etiquetado de alimentos" (food labeling), utilizándose como Major Topic en las bases que lo permitían, al garantizar la mayor sensibilidad de búsqueda y, por tanto, la consecución de los artículos más relevantes y pertinentes, eliminando de los resultados el ruido documental (artículos recuperados no relacionados con la temática principal de la búsqueda). Se utilizó el límite: humanos.

Las ecuaciones de búsqueda se desarrollaron para su empleo en la base de datos MEDLINE, vía PubMed, mediante la utilización de los conectores booleanos, adaptándose posteriormente a las otras bases de datos anteriormente mencionadas.

La búsqueda se realizó desde la primera fecha disponible, de acuerdo a las características de cada base de datos, hasta octubre de 2009 (momento de la última actualización).

\section{Selección final de los artículos}

La elección final de los trabajos se realizó según el cumplimiento de los criterios de inclusión y exclusión.

Criterios de inclusión: los documentos debían ser artículos originales publicados en revistas revisadas por pares. Igualmente, fueron 
seleccionados aquellos pertinentes cuyo texto completo pudo ser recuperado.

Criterios de exclusión: se eliminaron los artículos que no contenían resultados o símbolos de etiquetado, los que no habían sido estudiados en una población y aquellos que, aún versando sobre etiquetado de alimentos, su etiqueta no estaba dirigida a la prevención de la obesidad, sino a otros fines, como la prevención de patologías (por ejemplo: enfermedades cardiovasculares, diabetes, etc.).

Adicionalmente, como búsqueda secundaria y para reducir los posibles sesgos de publicación, se examinó el listado bibliográfico de los artículos que fueron seleccionados en la búsqueda principal, con el objeto de identificar estudios no detectados en la revisión.

Para determinar la pertinencia de los artículos se evaluaron de forma independientemente por dos de los autores de la presente revisión (M.I.S.-P. y J.S.-V.). Para dar por válida la elección de los artículos seleccionados para la revisión se estableció que la valoración de la concordancia entre estos dos autores (índice kappa) debía ser superior a 0,60 (fuerza de la concordancia buena o muy buena). Siempre que se cumpliera esta condición, las posibles discordancias se solucionarían mediante la consulta a la tercera autora (C.W.-B.) y posterior consenso entre todos los autores.

El control de calidad de la información se realizó mediante tablas de doble entrada que permitían la detección de los errores y la corrección mediante nueva consulta con los originales.

\section{Extracción de los datos}

Los estudios se agruparon con el objetivo de sistematizar y facilitar la comprensión de los resultados de todos los artículos revisados. Ningún artículo fue rechazado por causas metodológicas. No se establecieron restricciones en cuanto al género de los participantes, la edad o el tipo de muestra.

Todos los datos relevantes de cada trabajo se resumieron en una tabla, en concreto se codificaron los autores y año de publicación, la intervención efectuada, la población sometida al estudio, el periodo se seguimiento, el lugar donde se efectuó la intervención y las principales conclusiones señaladas en los artículos revisados.

Además, se determinaron los principales indicadores bibliométricos, entre ellos la obsolescencia medida por la mediana, también llamado Semiperiodo de Burton Kebler y el Índice de Price (porcentaje de referencias con edad igual o inferior a 5 años).

\section{Resultados}

De los 207 artículos recuperados, 53 (25,60\%) fueron de MEDLINE, 3 (1,45\%) de la Cochrane Library, 81 (39,13\%) de EMBASE, 59 (28,50\%) eran de la ISI Web of Knowledge y 11 (5,31\%) de Food Science and Technology Abstracts. No se obtuvieron resultados en las bases LILACS y CINAHL. Se identificaron 6 estudios más en los listados bibliográficos de los artículos recuperados. De los estudios encontrados, 58 (28,02\%) fueron artículos redundantes y $70(38,16 \%)$ no eran artículos originales.

Los datos descriptivos de los trabajos obtenidos en la búsqueda bibliográfica fueron: obsolescencia, medida por la mediana igual a 3 años (Índice de Burton Kebler), con un Índice de Price de 66,45\%. La mayoría de los trabajos, 53 (35,57\%), estaban firmados por un único autor. Siendo la principal institución productora la Universidad de Minnesota con 5 documentos (3,27\%). El país con mayor número de publicaciones sobre este tema fue Estados Unidos, con 81 artículos $(52,26 \%)$, aunque se denota una mayor preocupación, sobre el tema a estudio, en los países del ámbito anglosajón: Reino Unido con 24 (15,48\%) y Australia con 10 (6,45\%) artículos.

El idioma más utilizado fue el inglés, 150 artículos $(96,77 \%)$ y la revista con mayor número de trabajos publicados sobre esta temática fue la Journal of The American Dietetic Association, 10 artículos (6,49\%). El año con mayor número de artículos publicados fue el 2008, con 31 publicaciones $(20,00 \%)$. La mayor parte de los artículos, 137 (88,38\%), tenía acceso online; 95 $(61,68 \%)$ eran mediante pago y $41(27,27 \%)$ eran Open Access.

Del total de los 207 artículos se eliminaron 58 (28,02\%) al ser artículos redundantes encontrados en más de una de las bases de datos interrogadas. Además, 70 trabajos $(38,16 \%)$ no eran artículos originales, por lo que fueron eliminados. No cumplieron criterios de inclusión 17 trabajos $(8,21 \%)$ al no tratar los mismos sobre el tema a estudio (etiquetado relacionado con la obesidad en humanos). Posteriormente, 54 trabajos (26,09\%) se eliminaron al no ser intervenciones realizadas o probadas en población humana, o la intervención no estaba dirigida a la prevención de la obesidad o el sobrepeso, sino a otros fines como la prevención de patologías (enfermedades cardiovasculares, diabetes, cáncer, etc.)

Una vez aplicados los criterios de inclusión y exclusión (Figura 1), se seleccionaron 14 artículos 13,14,15,16,17,18,19,20,21,22,23,24,25,26 (Tabla 1). No fue necesaria la valoración de la concordancia entre los autores, ya que el acuerdo sobre la pertinencia de los trabajos fue del $100 \%$. 


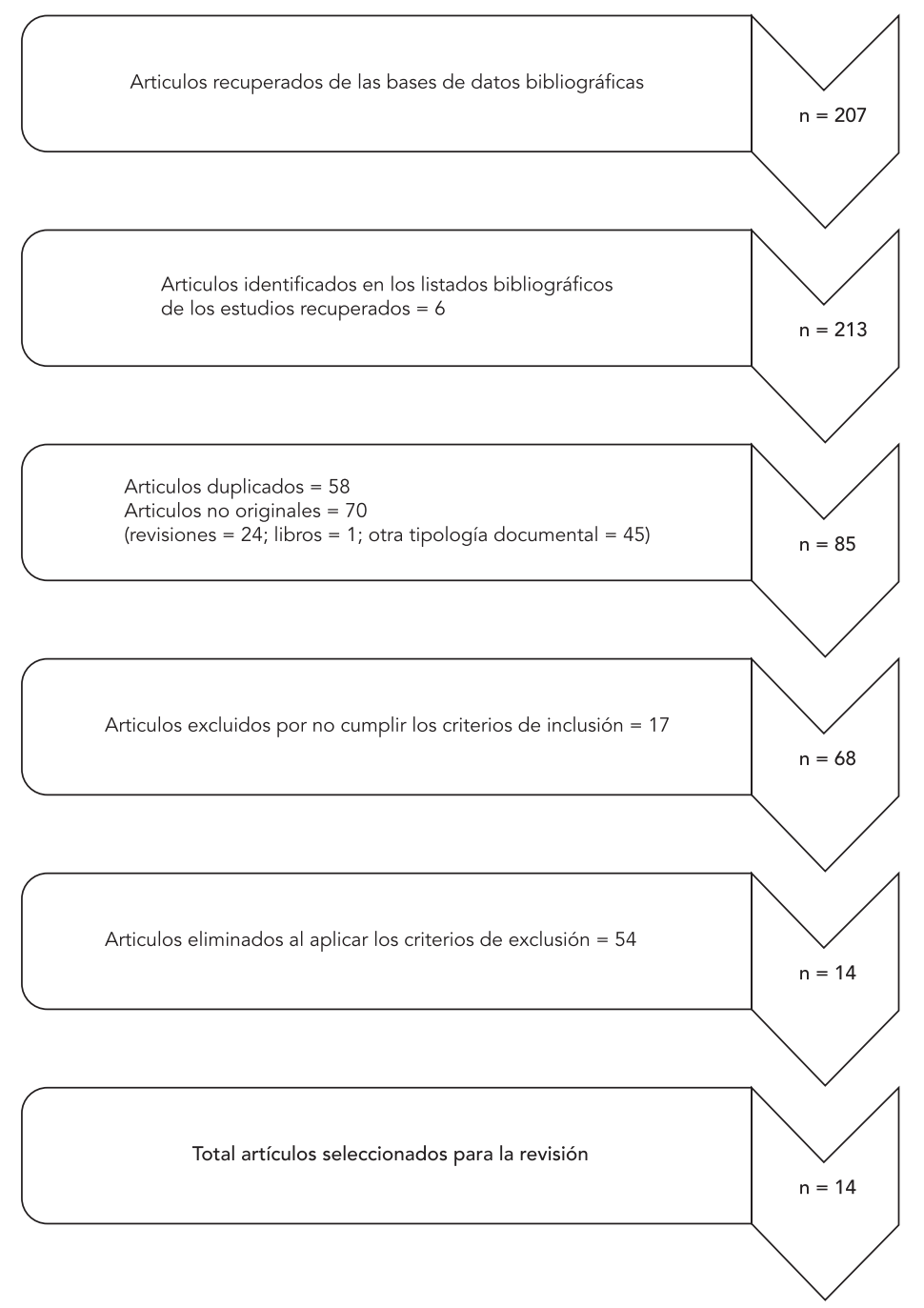

Los productos elegidos sobre los que observar su consumo y los resultados de la intervención fueron: refrescos 13 , golosinas 14, productos lácteos 15,26, platos de cafetería 16,17,20,21,23,24, productos de marca registrada 18 , comida rápida 19,25 y ensaladas 22. Los instrumentos más utilizados para la intervención fueron los pósteres motivadores y las etiquetas. La descripción detallada sobre los instrumentos utilizados para efectuar la intervención y los productos sobre los que se actuó, en relación al etiquetado y la rotulación en la prevención del sobrepeso y la obesidad se pueden consultar en la Tabla 2.
La información encontrada sobre la población sujeta a la intervención fue muy diversa: mientras en unos estudios se aporta información muy precisa sobre edad, género y etnia 18,19,24,26, en otro no se aportaban datos 22 . La intervención con mayor número de individuos tuvo una $\mathrm{n}=$ 5.24920 , siendo la de menor número de individuos de tan solo 43 personas 18 .

Los lugares en los que se llevaron a cabo las intervenciones fueron instituciones educativas $13,14,20,26$, laboratorios 15,18 , comedor militar 16 , comedor de hospital 17 , restaurantes $21,22,23,24$ y establecimientos de comida rápida 19,25. 
Resumen en orden cronológico de los 14 estudios revisados sobre etiquetado y rotulación de los alimentos para la prevención del sobrepeso y la obesidad, según el año de publicación.

\begin{tabular}{|c|c|c|c|c|c|c|}
\hline Referencia & Año & Intervención & Población & Periodo & Lugar & Conclusión \\
\hline Bergen et al. 13 & 2006 & $\begin{array}{l}\text { Instrumento: } \\
\text { etiquetas más } \\
\text { pósteres motivadores; } \\
\text { producto: refrescos } \\
\text { de máquinas } \\
\text { expendedoras }\end{array}$ & $\begin{array}{l}\text { Estudiantes, } \\
\text { profesores y plantilla } \\
\text { de personal }\end{array}$ & 5 semanas & $\begin{array}{l}\text { Colegio urbano } \\
\text { grande (EE.UU.) }\end{array}$ & $\begin{array}{l}\text { Las etiquetas que indican } \\
\text { las calorías y los pósteres } \\
\text { motivadores en las máquinas } \\
\text { expendedoras de bebida } \\
\text { pueden ser una manera eficaz } \\
\text { de influir en la venta } \\
\text { de bebidas }\end{array}$ \\
\hline Antonuk et al. 14 & 2006 & $\begin{array}{l}\text { Instrumento: } \\
\text { etiquetas de una sola } \\
\text { columna o de dos } \\
\text { columnas; producto: } \\
\text { golosinas }\end{array}$ & $\begin{array}{c}\mathrm{N}=112 ; \text { edad media }= \\
25,3 \text { años; sexo: } \\
\mathrm{H} / \mathrm{M}=50 / 62 ; \\
\text { mantienen una dieta }= \\
45 ; \text { no mantienen } \\
\text { una dieta }=67\end{array}$ & No consta & $\begin{array}{c}\text { Un curso de } \\
\text { introducción de } \\
\text { marketing en una } \\
\text { gran Universidad del } \\
\text { Noreste (EE.UU.) }\end{array}$ & $\begin{array}{l}\text { Participantes que no } \\
\text { mantienen una dieta comieron } \\
\text { significativamente menos } \\
\text { golosinas cuando fueron } \\
\text { expuestos a la etiqueta } \\
\text { de doble columna, en } \\
\text { comparación con la etiqueta } \\
\text { de una sola columna. } \\
\text { Participantes que mantenían } \\
\text { una dieta: (i) prestaron mayor } \\
\text { atención a la etiqueta; (ii) } \\
\text { percibían más la necesidad de } \\
\text { la etiqueta; y (iii) comprendían } \\
\text { mejor la información en la } \\
\text { etiqueta que los participantes } \\
\text { que no mantenían una dieta }\end{array}$ \\
\hline Roefs et al. 15 & 2004 & $\begin{array}{c}\text { Instrumento: } \\
\text { etiquetas con } \\
\text { contenido en grasa } \\
\text { (etiquetas de alto } \\
\text { contenido en grasa } \\
\text { [40\%] vs. etiquetas } \\
\text { con bajo contenido } \\
\text { en grasa [3\%]); } \\
\text { producto: batidos de } \\
\text { leche }\end{array}$ & $\begin{array}{c}\mathrm{N}=44 ; \text { edad: obesos } \\
(\mathrm{M}=34,0 \text { años } \\
\mathrm{SD}=4,9), \text { delgados } \\
(\mathrm{M}=33,9 \text { años } \\
\mathrm{SD}=8,5)\end{array}$ & No consta & Países Bajos & $\begin{array}{l}\text { El efecto de la etiqueta } \\
\text { fue el menor consumo del } \\
\text { producto etiquetado como } \\
\text { bajo en grasa y no hubo } \\
\text { diferencia entre grupos. } \\
\text { Inesperadamente, el orden } \\
\text { de presentación de los } \\
\text { productos (producto alto en } \\
\text { grasa primero vs. producto } \\
\text { bajo en grasa primero) tuvo } \\
\text { un gran efecto en el consumo }\end{array}$ \\
\hline Sproul et al. 16 & 2003 & $\begin{array}{l}\text { Instrumento: } \\
\text { etiquetas, pósteres, } \\
\text { folletos e imanes } \\
\text { para refrigerador; } \\
\text { producto: } 43 \text { platos } \\
\text { principales }\end{array}$ & $\begin{array}{c}\mathrm{n}=149 ; \text { edad: } \mathrm{M}= \\
28 \text { años; sexo: } \mathrm{H} / \mathrm{M}= \\
\text { 132/13; } \text { Missing }=4\end{array}$ & 15 meses & $\begin{array}{c}\text { Un servicio de } \\
\text { comedor del Ejército } \\
\text { (EE.UU.) }\end{array}$ & $\begin{array}{l}\text { Una campaña de marketing } \\
\text { basada en las propiedades } \\
\text { saludables de los platos } \\
\text { principales no tiene influencia } \\
\text { en la elección de estos } \\
\text { platos. Parece que los } \\
\text { atributos sensoriales son más } \\
\text { determinantes }\end{array}$ \\
\hline Milich et al. 17 & 1976 & $\begin{array}{l}\text { Instrumento: } \\
\text { presentación de } \\
\text { calorías en tarjetas; } \\
\text { producto: todos } \\
\text { los artículos en una } \\
\text { cafetería, excepto } \\
\text { los refrescos y los } \\
\text { condimentos }\end{array}$ & $\begin{array}{c}\mathrm{n}=450+(\mathrm{n}=150 \\
\text { peso normal; } \mathrm{n}=150 \\
\text { sobrepeso; } \mathrm{n}=150 \\
\text { obesas }\end{array}$ & 4 semanas & $\begin{array}{l}\text { La cafetería principal } \\
\text { en North Carolina } \\
\text { Memorial Hospital, } \\
\text { Chapel Hill, Carolina } \\
\text { del Norte (EE.UU.) }\end{array}$ & $\begin{array}{l}\text { La presentación de los } \\
\text { valores calóricos de los } \\
\text { alimentos podría disminuir } \\
\text { significativamente el número } \\
\text { total de calorías adquiridas } \\
\text { por personas de todos los } \\
\text { grupos de peso }\end{array}$ \\
\hline
\end{tabular}

(continua) 
Tabla 1 (continuación)

\begin{tabular}{|c|c|c|c|c|c|c|}
\hline Referencia & Año & Intervención & Población & Periodo & Lugar & Conclusión \\
\hline Forman et al. 18 & 2009 & $\begin{array}{l}\text { Instrumento: alimentos } \\
\text { con marca y alimentos } \\
\text { sin marca; producto: } 30 \\
\text { productos distintos }\end{array}$ & $\begin{array}{c}\mathrm{n}=43 ; \text { edad (años): } \\
\text { 4-6 años; } M=5,9 \\
\text { años }( \pm 0,9) \text {; sexo: } \\
\text { H/M = 17/26; etnia: } \\
\text { afroamericano = 16, } \\
\text { hispano }=13 \\
\text { caucásico }=7, \text { asiático = } \\
\text { 2, otros }=5 ; n=23 \text { sin } \\
\text { sobrepreso; } n=20 \text { con } \\
\text { sobrepeso }\end{array}$ & $\begin{array}{l}4 \text { cenas de } 30 \\
\text { minutos: dos } \\
\text { con productos } \\
\text { con marca } \\
\text { y dos con } \\
\text { productos sin } \\
\text { marca }\end{array}$ & $\begin{array}{l}\text { Laboratorio situado en } \\
\text { Roosevelt St. Luke's } \\
\text { Hospital, Nueva York, NY } \\
\text { (EE.UU.) }\end{array}$ & $\begin{array}{l}\text { Los niños con sobrepeso } \\
\text { consumieron más alimentos con } \\
\text { marca y pueden estar en riesgo } \\
\text { en los ambientes que están muy } \\
\text { inundados con mensajes acerca } \\
\text { de los alimentos }\end{array}$ \\
\hline Harnack et al. 19 & 2008 & $\begin{array}{l}\text { Instrumento: etiquetas } \\
\text { con información } \\
\text { calórica y precio de } \\
\text { la porción; cuadro } \\
\text { de información } \\
\text { sobre "necesidades } \\
\text { energéticas diarias } \\
\text { por sexo"; producto: } \\
\text { comida rápida }\end{array}$ & $\begin{array}{c}\text { n= 594; edad (años): } \\
M=40,84 \text { años; sexo: } \\
\text { H/M = 241/353; etnia: } \\
\text { hispano/latino = 20, } \\
\text { No hispano/latino = } \\
\text { 567; IMC: normopeso = } \\
\text { 249, sobrepeso }=163, \\
\text { obeso }=173\end{array}$ & 7 meses & $\begin{array}{c}\text { Instalaciones de } \\
\text { McDonald's (EE.UU.) }\end{array}$ & $\begin{array}{l}\text { No se encontraron diferencias } \\
\text { entre la composición } \\
\text { energética de los menús } \\
\text { consumidos. Los resultados } \\
\text { estratificados por edad, etnia, } \\
\text { peso o nivel cultural también } \\
\text { fueron similares }\end{array}$ \\
\hline Snelling et al. 20 & 2007 & $\begin{array}{l}\text { Instrumento: etiquetar } \\
\text { alimentos en base a su } \\
\text { densidad nutricional; } \\
\text { producto: todos } \\
\text { los productos en la } \\
\text { cafetería }\end{array}$ & $\begin{array}{c}\mathrm{n}=5.249 ; \text { etnia: } \\
\text { blancos }=2.102, \\
\text { negros }=855, \\
\text { hispanos }=1.698, \\
\text { asio-americanos }=578, \\
\text { Otros }=21\end{array}$ & 4 semanas & $\begin{array}{c}\text { Cafeterías de } 3 \\
\text { escuelas de secundaria } \\
\text { públicas (EE.UU.) }\end{array}$ & $\begin{array}{c}\text { Los estudiantes compran } \\
\text { alimentos con mínimo valor } \\
\text { nutricional en las cafeterías de } \\
\text { los colegios }\end{array}$ \\
\hline Mayer et al. 21 & 1986 & $\begin{array}{l}\text { Instrumento: pósteres } \\
\text { y folletos; producto: } \\
\text { platos principales bajos } \\
\text { en grasa }\end{array}$ & $\begin{array}{c}N=3.264 ; \text { sexo: } \\
\text { H/M: } 1\end{array}$ & 9 semanas & $\begin{array}{l}\text { Cafetería cerca de } \\
\text { campus en St. Louis, } \\
\text { Missouri (EE.UU.) }\end{array}$ & $\begin{array}{l}\text { La intervención produjo un } \\
\text { incremento significativo (del } \\
20 \% \text { al } 35 \% \text { ) en la venta de } \\
\text { los platos principales bajos en } \\
\text { calorías }\end{array}$ \\
\hline Wagner et al. 22 & 1988 & $\begin{array}{c}\text { Instrumento: pósteres, } \\
\text { tarjetas de mesa; } \\
\text { producto: ensaladas }\end{array}$ & No consta & 11 semanas & $\begin{array}{l}\text { Dos restaurantes } \\
\text { de comida rápida } \\
\text { en Blacksburg, y en } \\
\text { Radford, Virginia } \\
\text { (EE.UU.) }\end{array}$ & $\begin{array}{l}\text { El estudio muestra que } \\
\text { una mínima inversión en } \\
\text { instrumentos (pósteres y } \\
\text { tarjetas de mesa) podrían } \\
\text { aumentar el consumo de } \\
\text { ensaladas en los menús }\end{array}$ \\
\hline Dubbert et al. 23 & 1984 & $\begin{array}{l}\text { Instrumento: etiquetas, } \\
\text { pósteres; producto: } \\
\text { productos bajos en } \\
\text { grasa }\end{array}$ & $\begin{array}{c}N=413 ; \text { Sexo: } H / M= \\
187 / 226\end{array}$ & 15 semanas & Cafetería (EE.UU.) & $\begin{array}{c}\text { Intervenciones ambientales } \\
\text { pueden ser efectivas en los } \\
\text { cambios de compra de comida } \\
\text { en las cafeterías, pero el } \\
\text { etiquetado individual puede } \\
\text { que no sea un método más } \\
\text { efectivo para disminuir la } \\
\text { compra total de calorías }\end{array}$ \\
\hline Levin 24 & 1996 & $\begin{array}{l}\text { Instrumento: póster, } \\
\text { etiquetas en forma de } \\
\text { corazón; producto: } \\
\text { productos bajos en } \\
\text { grasa }\end{array}$ & $\begin{array}{c}\mathrm{N}=138 \text {; edad: } M= \\
41 \text { años; sexo: } \mathrm{H} / \mathrm{M}= \\
46 / 92 \text {; etnia: } \\
\text { hispanos }=70, \text { blancos } \\
\quad=53, \text { negros }=8, \\
\text { nativos americanos }=8, \\
\text { asiáticos }=3, \text { otros }=4 \\
\text { (8 personas pertenecían } \\
\text { a más de una categoría) }\end{array}$ & $\begin{array}{l}6 \text { semanas; } \\
\text { seguimiento } \\
\text { de dos } \\
\text { semanas a los } \\
7 \text { meses }\end{array}$ & $\begin{array}{c}\text { Dos cafeterías en } \\
\text { Albuquerque, Nuevo } \\
\text { México (EE.UU.) }\end{array}$ & $\begin{array}{l}\text { La intervención produce } \\
\text { un aumento en el consumo } \\
\text { de los platos principales } \\
\text { bajos en grasa. La venta de } \\
\text { estos productos continúa } \\
\text { a los } 7 \text { meses. Este tipo } \\
\text { de intervención puede ser } \\
\text { especialmente útil en grupos } \\
\text { con bajo nivel cultural }\end{array}$ \\
\hline
\end{tabular}

(continua) 
Tabla 1 (continuación)

\begin{tabular}{|c|c|c|c|c|c|c|}
\hline Referencia & Año & Intervención & Población & Periodo & Lugar & Conclusión \\
\hline $\begin{array}{l}\text { Yamamoto } \\
\text { et al. } 25\end{array}$ & 2004 & $\begin{array}{l}\text { Instrumento: menús } \\
\text { informando de la } \\
\text { cantidad de grasa y } \\
\text { calorías; producto: } \\
3 \text { menús de comida } \\
\text { rápida }\end{array}$ & $\begin{array}{c}N=106 ; \text { Edad (años): } \\
M=14,4 \pm 2,0 ; \text { Sexo: } \\
\text { H/M }=51 / 55\end{array}$ & No consta & $\begin{array}{l}3 \text { cadenas de } \\
\text { restaurantes de comida } \\
\text { rápida (EE.UU.) }\end{array}$ & $\begin{array}{l}\text { Aportar información en calorías } \\
\text { y grasa en los menús no } \\
\text { afecta al consumo final de los } \\
\text { adolescentes, sin embargo, } \\
\text { esta información podría ayudar } \\
\text { a otros adolescentes y no } \\
\text { supone una reducción en los } \\
\text { ingresos de los restaurantes }\end{array}$ \\
\hline $\begin{array}{l}\text { Wechsler } \\
\text { et al. } 26\end{array}$ & 1998 & $\begin{array}{l}\text { Instrumento: } \\
\text { pósteres con "Lowfat } \\
\text { Lucy". auditorio, } \\
\text { juegos interactivos, } \\
\text { personaje "Lowfat } \\
\text { Lucy" concurso } \\
\text { de rompecabezas, } \\
\text { premios, folletos; } \\
\text { Producto: leche baja } \\
\text { en grasa }\end{array}$ & $\begin{array}{l}\mathrm{N}=6.902 ; \text { Control } \\
\text { (n = 2.840; Etnia: } \\
\text { hispanos = 2.319, } \\
\text { afro-americanos = } \\
\text { 383, otros); } \\
\text { intervención } \\
\text { (n = 4.062; etnia: } \\
\text { hispanos = 3.560, } \\
\text { afro-americanos = } \\
337, \text { otros) }\end{array}$ & $\begin{array}{l}60 \text { días. } \\
\text { Seguimiento } \\
\text { a los 3-4 } \\
\text { meses, } \\
\text { durante } 5 \\
\text { días }\end{array}$ & $\begin{array}{c}6 \text { escuelas primarias } \\
\text { públicas ( } 3 \text { control, } 3 \\
\text { intervención) de bajos } \\
\text { ingresos, en el centro } \\
\text { de la ciudad, en zona } \\
\text { de la comunidad latina } \\
\text { en Nueva York } \\
\text { (EE.UU.) }\end{array}$ & $\begin{array}{l}\text { El consumo de leche baja en } \\
\text { grasa se incrementó en los } \\
\text { colegios con intervención } \\
\text { del } 25 \% \text { al } 57 \% \text {, pero } \\
\text { permaneció constante en } \\
\text { los colegios control en } \\
\text { un } 28 \% \text {. Las diferencias } \\
\text { entre los colegios control e } \\
\text { intervención permanecieron } \\
\text { siendo significativas en el } \\
\text { seguimiento, 3-4 meses } \\
\text { después }\end{array}$ \\
\hline
\end{tabular}

Tabla 2

Instrumentos utilizados para efectuar la intervención y productos sobre los que se ha intervenido en orden cronológico, referentes al etiquetado y rotulación de los alimentos, en la prevención del sobrepeso y la obesidad, según el año de publicación.

\begin{tabular}{|c|c|c|c|}
\hline Referencia & Año & Intervención & Descripción de la intervención \\
\hline Bergen et al. 13 & 2006 & $\begin{array}{l}\text { Instrumento: etiquetas más pósteres motivacionales; } \\
\text { producto: refrescos de máquinas expendedoras }\end{array}$ & $\begin{array}{c}\text { Etiquetas en los paneles de selección con colores brillantes } \\
\text { "0 calorías, } 0 \text { carbohidratos"; pósteres motivadores } \\
\text { promocionando agua y refrescos sin calorías }\end{array}$ \\
\hline Antonuk et al. 14 & 2006 & $\begin{array}{l}\text { Instrumento: etiquetas de una sola columna o de dos } \\
\text { columnas; producto: golosinas }\end{array}$ & $\begin{array}{l}\text { La etiqueta con una columna de información nutricional sobre } \\
\text { una porción del producto y la etiqueta de doble columna que } \\
\text { refleja la información nutricional de una porción, así como la } \\
\text { información para el paquete entero }\end{array}$ \\
\hline Roefs et al. 15 & 2004 & $\begin{array}{l}\text { Instrumento: etiquetas con contenido en grasa } \\
\text { (etiquetas de alto contenido en grasa [40\%] vs. } \\
\text { etiquetas con bajo contenido en grasa [3\%]); } \\
\text { producto: batidos de leche }\end{array}$ & $\begin{array}{l}\text { Dos etiquetas " } 40 \% \text { de grasa" en una sesión; dos etiquetas } \\
\text { "3\% de grasa" en otra sesión para el mismo producto }\end{array}$ \\
\hline Sproul et al. 16 & 2003 & $\begin{array}{l}\text { Instrumento: etiquetas, pósteres, folletos e imanes } \\
\text { para refrigerador; producto: } 43 \text { platos principales }\end{array}$ & $\begin{array}{l}\text { Etiquetas: una por producto, en la línea de servicio, con el } \\
\text { logo promocional (rayo rojo encastrado en un cuadrado azul). } \\
\text { Las calorías, las grasas y el colesterol también aparecían en } \\
\text { esta etiqueta, en color negro; pósteres: grandes y de colores, } \\
\text { en las paredes del centro y a la entrada del comedor, con } \\
\text { el logo promocional y el eslogan promocional (“Es un signo } \\
\text { seguro de que está comiendo mejor ); folletos: de una página, } \\
\text { con la información nutricional, a la entrada del comedor; imanes } \\
\text { para refrigerador con el logo promocional }\end{array}$ \\
\hline Milich et al. 17 & 1976 & $\begin{array}{l}\text { Instrumento: presentación de calorías en tarjetas; } \\
\text { producto: todos los artículos en una cafetería, } \\
\text { excepto los refrescos y los condimentos }\end{array}$ & $\begin{array}{l}\text { Etiquetas con el nombre del producto y el valor calórico en } \\
\qquad \text { tinta roja }\end{array}$ \\
\hline
\end{tabular}

(continua) 
Tabla 2 (continuación)

\begin{tabular}{lcr}
\hline Referencia & Año & Intervención \\
\hline Forman et al. 18 & 2009 & $\begin{array}{r}\text { Instrumento: alimentos con marca y alimentos sin } \\
\text { marca; producto: } 30 \text { productos distintos }\end{array}$ \\
Harnack et al. 19 & 2008 & $\begin{array}{r}\text { Instrumento: etiquetas con información calórica } \\
\text { y precio de la porción; cuadro de información } \\
\text { sobre "necesidades energéticas diarias por sexo"; } \\
\text { producto: comida rápida }\end{array}$
\end{tabular}

Snelling et al. 20

Mayer et al. 21

Wagner et al. 22

Instrumento: póster, etiquetas en forma de corazón; producto: productos bajos en grasa

Instrumento: etiquetar alimentos en base a su densidad nutricional; producto: todos los productos en la cafetería

Instrumento: pósteres y folletos; producto: platos principales bajos en grasa

Instrumento: pósteres, tarjetas de mesa; producto: ensaladas

Instrumento: etiquetas, pósteres; producto: productos bajos en grasa

Instrumento: menús informando de la cantidad de grasa y calorías; producto: 3 menús de comida rápida

Instrumento: pósteres con "Lowfat Lucy". auditorio, juegos interactivos, personaje "Lowfat Lucy" concurso de rompecabezas, premios, folletos; producto: leche baja en grasa

\section{Descripción de la intervención}

Productos en su envase original mostrando su marca; los mismos productos, en envases transparentes y sin identificar

$$
\text { con su marca }
$$

Etiqueta en cuatro versiones: (i) sin información de precio o calorías, (ii) con información de precio, (iii) con información de calorías, y (iv) con información de precio y calorías; cuadro de información sobre las necesidades energéticas diarias por sexo

Etiqueta semáforo. 3 categorías: (i) Alimentos verdes: bajos en calorías, (ii) alimentos amarillos: moderados en calorías, y (iii) alimentos rojos: altos en calorías

Póster grande y a color, con el listado de beneficios de la dieta baja en grasas y los platos principales del día bajos en grasa , al principio de la línea de servicio; 2 pósteres más pequeños con los platos principales bajos en calorías, en el lugar de los platos principales; folletos pequeños con el mismo mensaje del póster en cada mesa

2 pósteres grandes a color con el mensaje:"Estar en forma y saludable; come una ensalada baja en grasa como plato principal o agrega una ensalada" y un dibujo de una ensalada cerca de la caja registradora; tarjetas en cada mesa con el mismo mensaje que en el póster; decoración a la entrada con el mensaje "Come ensaladas"

Etiquetas identificando los productos bajos en grasa. Etiquetas de papel verde brillante con letras en negro con el mensaje: "SELECCIÓN BAJA EN CALORIAS", y un punto rojo brillante; póster en verde, con letras negras y leyenda:"PARA SU INFORMACIÓN, HEMOS ETIQUETADO ALGUNOS PRODUCTOS BAJOS EN GRASA.... Observe estos signos"; un póster igual y más pequeño al principio de la línea de servicio

Un póster a la entrada de la cafetería con la leyenda:"busca para tu selección de plato principal bajo en grasas"; etiquetas con formas de corazón junto al plato principal específico en el tablero del menú

Menús acompañados con información sobre calorías y contenido en grasa

Pósteres: colgados en las paredes de los centros y actualizados para la fecha en que iba a tener lugar la intervención, con el propósito de la expectación; auditorio, informando sobre los beneficios del consumo de la leche baja en grasa; concurso de rompecabezas, juegos interactivos, premios (camisetas con el eslogan "Si tienes más de dos años, la leche baja en grasa es mejor para ti"); el personaje de "Lowfat Lucy", una vaca antropométrica estilo Disney; folletos para informar a los padres de forma sencilla sobre los beneficios de la leche baja en grasa 
Los estudios no seleccionaron por género, excepto en el comedor hospitalario 17, que sólo incluyó mujeres en la intervención, $\mathrm{n}=450$, clasificadas en tres grupos dependiendo de su peso.

El periodo de estudio más largo se llevó a cabo en el comedor militar 16: 15 meses. Por el contrario, el que menor tiempo ocupó fue el trabajo referente a la información puntual sobre golosinas 14 .

Resaltar que 13 de las 14 intervenciones tuvieron lugar en Estados Unidos y tan solo una se desarrolló en los Países Bajos 15.

La mayoría de los estudios concluyeron que las intervenciones realizadas tenían efecto en el consumo final de producto $13,14,15,17,19,20,21,22,23$, 24,26. Si bien, en los establecimientos de comida rápida, la intervención que se realizó no tuvo efecto en el consumo final 19,25. En el comedor militar, los atributos sensoriales pesaron más que las recomendaciones saludables 16. Significar que en dos estudios seguimientos posteriores confirmaron el efecto a largo plazo de las intervenciones realizadas 24,26 .

\section{Discusión}

El estudio de la actualidad/obsolescencia de los trabajos revisados demuestra que el tema seleccionado es de plena vigencia, ya que 2 de cada 3 artículos han sido publicados en los últimos 5 años 27. Los restantes resultados del análisis bibliométrico de la producción científica revisada muestran unos datos similares a los ofrecidos por trabajos previos en el entorno de las ciencias de la salud 28,29,30.

Destacar el hecho de que la institución con mayor número de estudios sobre la temática sea la Universidad de Minnesota. Esta Universidad tiene una larga y excelente trayectoria en los estudios sobre la nutrición en la prevención de la enfermedad, enfatizando el estudio sobre dieta mediterránea, The Seven Countries Study, efectuado por Ancel Keys et al. (http://www.sph. umn.edu/epi/history/sevencountries.asp).

Posibles limitaciones o particularidades del estudio, puesto que, pese a que es preferible basar una revisión sistemática en estudios con periodos de seguimiento adecuados, así como circunscribir la revisión a trabajos con un apropiado diseño que garanticen la evidencia científica de las conclusiones finales, se decidió incluir todos los trabajos recuperados y considerados como pertinentes, con la única condición de haber efectuado una intervención poblacional sobre repercusiones del etiquetado o la rotulación de los alimentos.
En este mismo sentido, no se, incluso en algunos trabajos no se aportaban datos sobre la misma. limitó el tamaño de la población para poder abarcar el máximo número de artículos posibles, incidiendo, como ya se ha comentado, en la intervención efectuada. Queda patente, que la población existente en los estudios revisados presentaba datos muy diversos en relación con la edad, género o etnia

La mayoría de los estudios concluyeron que las intervenciones (pósteres, etiquetas) realizadas, tenían efecto en el consumo final de producto. Sin embargo, las etiquetas no ofrecen una buena fiabilidad de manera universal: países como Brasil muestran altos índices de falta de fiabilidad en los datos de las etiquetas de los alimentos dirigidos a niños y adolescentes, dato destacado en un trabajo anterior 31 . Numerosos estudios avalan que mostrar el número de calorías de los productos en restaurantes y cafeterías, junto con los mensajes promocionales, tienen un efecto muy positivo a la hora de disminuir el número de calorías consumidas 32 . Además, poblaciones definidas, como los estudiantes, afirman que quieren observar el etiquetado nutricional para poder tomar decisiones a la hora de la compra ${ }^{33}$. La manera en que se realizan las intervenciones es importante; en el caso del etiquetado con la leyenda "bajo en grasas", el consumidor percibe una falsa sensación de disminución del número de calorías en el producto y, a la vez, se produce una disminución del sentimiento de culpabilidad a la hora de consumirlo, y sin embargo, "bajo en grasas", no quiere decir bajo en calorías y se conoce que hay ácidos grasos que son esenciales 34 . En otros estudios, se defiende que en ciertos grupos, para que las intervenciones tengan efecto, se ha de actuar directamente sobre cada individuo de la población, porque de otra manera, no se consigue ningún resultado: es el caso que exponen Jain et al. 35 en el que madres con bajos ingresos no se preocupan por el sobrepeso de sus hijos, aun siendo informadas en las etiquetas de los productos de las necesidades nutricionales de sus niños. En esta ocasión, los autores defienden que se ha de llegar directamente a la madre con la intervención de los profesionales, los pediatras, porque la información en la etiqueta no llega a ser comprendida por ellas.

En los establecimientos de comida rápida donde se realizó la intervención, no hubo efecto en el consumo final de productos. Es más, hay evidencias positivas de la correlación entre el consumo de comida rápida y el incremento de energía ingerida ${ }^{36}$, existiendo muchas y variadas razones por las que ir a los restaurantes de comida rápida, según el trabajo de Rydell et al. ${ }^{37}$. No obstante, la elección de la comida en los fast-food 
viene determinada sobre todo por el sabor de los alimentos 38. Catherine Adams 39, vicepresidenta de McDonald's, defiende la comida rápida indicando que se informa de forma adecuada sobre las calorías y la composición nutricional de estos productos, tanto en carteles promocionales en los establecimientos, como en los manteles de las bandejas de los menús. También señala que la materia prima que se utiliza en estos productos es de primera calidad.

Los atributos sensoriales en los comedores militares pesaron más, según los datos hallados en esta revisión, que las recomendaciones saludables. Está claro que el sabor no es negociable y habría que combatir la percepción que existe sobre los alimentos saludables de su falta de creatividad y aroma 40 . Además, a esto hay que añadir, que cuando compras un dulce, por un poco más de dinero tienes una porción el doble de grande. En este punto, las emociones y las sensaciones también tienen mucho que ver en las decisiones de consumo 7 .

Otro problema que está surgiendo actualmente es la asociación que se está realizando entre alimento divertido (fun food), que suele coincidir con productos artificiales, con poca calidad nutricional, y alimentación. Todo esto valiéndose de imágenes evocadoras y conocidas; sin percatarse, o incluso haciéndolo, que alimentarse no es un juego ${ }^{41}$.

En relación a los seguimientos posteriores, en los artículos que ofrecieron este dato, notificaron la continuidad del efecto a largo plazo. Es por eso, que las acciones sobre el etiquetado deberían ir acompañadas de talleres de educación para promover su uso y comprensión 42 , aunque esta circunstancia tendría que ser mejor estudiada y comprobada en los trabajos sobre intervención poblacional.

Etiquetar de forma entendible, aprender cómo leer una etiqueta, además de otras intervenciones (traffic light), podrían tener un papel decisivo en el cambio de comportamiento del consumidor 43. En muchos de los artículos revisados se demandan campañas de educación para interpretar las etiquetas de forma que pueda ayudarse a la población a realizar elecciones saludables en sus comidas, destacando el trabajo de Krukonski et al. ${ }^{44}$. No obstante, los intentos de lograr "el consumidor informado" con la esperanza de que pueda elegir de forma más saludable no han logrado un éxito demostrable, siendo poco probable el cambio de comportamiento de los consumidores, no alcanzándose la respuesta deseada en la prevención de la obesidad y de las enfermedades relacionadas con la dieta 45 . A propósito de esta demanda, se están creando instituciones como el Obesity Working Group (OWG), avalado por la International Association for the Study of Obesity (IASO), que investiga el comportamiento de los consumidores y busca nuevos enfoques para favorecer las alternativas saludables (http:// www.iaso.org/site_media/uploads/IASO_Sum mary_Report_2009.pdf). Está claro que la educación en salud es importante y por eso desde la escuela hay que aprovechar la oportunidad de poder incidir sobre la creciente incidencia de sobrepeso en niños y adolescentes: los niños pasan gran parte de su tiempo en la escuela y crear un ambiente escolar saludable puede influir en los hábitos alimenticios y físicos de los niños; los hombres del futuro ?.

La aparición con la obesidad y el sobrepeso de una mayoría de estudios sobre intervención poblacional, relacionada con el etiquetado y la obesidad en Estados Unidos, puede deberse a la alta prevalencia de la obesidad y el sobrepeso en esta población. La preocupación que existe en este país sobre la temática a estudio puede verse en la página del Centers for Disease Control and Prevention (http://www.cdc.gov/obesity/ childhood/data.html), donde se observa que en algunos Estados más de 2 de cada 10 niños sufre sobrepeso $\mathrm{u}$ obesidad (2009 State Prevalence Among Low-Income Children Aged 2 to 4 Years) y 1 de cada 3 niños, pertenecientes a familias con bajos ingresos, presenta ya esta enfermedad. En esta situación, es lógica la preocupación por los estudios poblacionales relacionados

Por todo lo anteriormente expuesto, se puede concluir:

- Las intervenciones en el etiquetado y la rotulación suelen tener efecto positivo en el consumo final de los alimentos;

- Esta circunstancia no se observa en los restaurantes de comida rápida;

- Los atributos sensoriales fueron más efectivos que las recomendaciones recogidas en el etiquetado de los productos;

- El seguimiento de las actuaciones, en los artículos que ofrecieron este dato, notificaron la continuidad del efecto a largo plazo de las intervenciones estudiadas. 


\section{Resumen}

Revisión de los artículos sobre el etiquetado o la rotulación en la prevención de la obesidad y el sobrepeso. Estudio, mediante técnica sistemática, de los trabajos recuperados mediante revisión bibliográfica. Bases de datos consultadas: MEDLINE, EMBASE, Web of Knowledge, Cochrane Library Plus, Food Science and Technology Abstracts, LILACS y CINAHL. Descriptores DeCS/MeSH: obesity y food labeling. 207 artículos recuperados, mediante los criterios de inclusión y exclusión, se seleccionaron 14: 11 sobre etiquetado y la rotulación en el consumo final del producto; 2 sobre establecimientos de comida rápida. Uno sobre atributos sensoriales en contraste con recomendaciones saludables y 2 realizaron un seguimiento de las intervenciones. El etiquetado y la rotulación tienen efecto positivo en el consumo final de los alimentos, suceso no observado en restaurantes de comida rápida. Los atributos sensoriales fueron más efectivos que las recomendaciones recogidas en el etiquetado. El seguimiento de las actuaciones confirmó el efecto a largo plazo de las intervenciones estudiadas.

Etiquetado de Alimentos; Obesidad; Política Nutricional; Hábitos Alimenticios; Conducta Alimentaria

\section{Colaboradores}

M. I. Sebastián-Ponce contribuyó con la concepción y diseño del estudio, adquisición de datos, análisis de datos, interpretación de los datos y redacción del manuscrito. J. Sanz-Valero contribuyó con la concepción y diseño del estudio, análisis de datos, interpretación de los datos, redacción del manuscrito y aprobación definitiva. C. Wanden-Berghe contribuyó con el análisis de datos, interpretación de los datos, redacción del manuscrito y aprobación definitiva.

\section{Referencias}

1. Hyde R. Europe battles with obesity. Lancet 2008; 371:2160-1.

2. Kersh R. The politics of obesity: a current assessment and look ahead. Milbank Q 2009; 87:295-316.

3. Stanton R. Who will take responsibility for obesity in Australia? Public Health 2009; 123:280-2.

4. Magnusson RS. What's law got to do with it? Part 2: legal strategies for healthier nutrition and obesity prevention. Aust New Zealand Health Policy 2008; 5:e11.

5. Lachat C, Roberfroid D, Huybregts L, Van Camp J, Kolsteren P. Incorporating the catering sector in nutrition policies of WHO European Region: is there a good recipe? Public Health Nutr 2009; 12:316-24.

6. Mello MM, Studdert DM, Brennen TA. Obesity: the new frontier of public health law. $N$ Engl J Med 2006; 106:2601-10.

7. Hayne CL, Moran PA, Ford MM. Regulating environments to reduce obesity. J Public Health Policy 2004; 25:391-407.

8. Berman M, Lavizzo-Mourey R. Obesity prevention in the information age: caloric information at the point of purchase. JAMA 2008; 300:433-5.
9. Philipson T. Government perspective: food labeling. Am J Clin Nutr 2005; 82(1 Suppl):262S-4S.

10. Meadows M. The FDA forms obesity working groups. FDA Consum 2004; 38:28-9.

11. Switt J. Labeling around the globe: helping to direct food flow. J Am Diet Assoc 2007; 107:199-200.

12. Bruce A. Strategies to prevent the metabolic syndrome at the population level: role of authorities and non-governmental bodies. Br J Nutr 2000; 83(1 Suppl):181S-6S.

13. Bergen D, Yeh MC. Effects of energy-content labels and motivational posters on sales of sugarsweetened beverages: stimulating sales of diet drinks among adults study. J Am Diet Assoc 2006; 106:1866-9.

14. Antonuk B, Block LG. The effect of single serving versus entire packaged nutritional information on consumption norms and actual consumption of a snack food. J Nutr Educ Behav 2006; 38:365-70.

15. Roefs A, Jansen A. The effect of information about fat content on food consumption in overweight/ obese and lean people. Appetite 2004; 43:319-22. 
16. Sproul AD, Canter DD, Schmidt JB. Does point-of purchase nutrition labeling influence meal selections? A test in an Army cafeteria. Mil Med 2003; 168:556-60.

17. Milich R, Anderson J, Mills M. Effects of visual presentation of caloric values on food buying by normal and obese persons. Percept Mot Skills 1976; 42:155-62.

18. Forman J, Halford JCG, Summe H, MacDougall M, Keller KL. Food branding influences ad libitum intake differently in children depending on weight status. Result of a pilot study. Appetite 2009; 53: 76-83.

19. Harnack LJ, French SA, Oakes JM, Story MT, Jeffery RW, Rydell SA. Effects of calorie labeling and value pricing on fast food meal choices: results from a experimental trial. Int J Behav Nutr Phys Act 2008; 5:63.

20. Snelling AM, Korba C, Burkey A. The National School Lunch and competitive food offerings and purchasing behaviors of high school students. J Sch Health 2007; 77:701-5.

21. Mayer JA, Heins JM, Vogel JM, Morrison DC, Lankester LD, Jacobs AL. Promoting low-fat entree choices in a public cafeteria. J Appl Behav Anal 1986; 19:397-402.

22. Wagner JL, Winett RA. Prompting one low-fat, high-fiber selection in a fast-food restaurant. J Appl Behav Anal 1988; 21:179-85.

23. Dubbert PM, Johnson WG, Schlundt DG, Montague NW. The influence of caloric information on cafeteria food choices. J Appl Behav Anal 1984; 17:85-92.

24. Levin S. Pilot study of a cafeteria program relying primarily on symbols to promote healthy choices. J Nutr Educ 1996; 28:282-5.

25. Yamamoto JA, Yamamoto JB, Yamamoto BE, Yamamoto LG. Adolescent fast food and restaurant ordering behavior with and without calorie and fat content menu information. J Adolesc Health 2005; 37:397-402.

26. Wechsler H, Basch CE, Zybert P, Shea S. Promoting the selection of low-fat milk in elementary school cafeterias in an inner-city latino community: evaluation of an intervention. Am J Public Health 1998; 88:427-33.

27. Culebras-Fernández J, García de Lorenzo A, Wanden-Berghe C, Castiel LD, Sanz-Valero J. Careful! Your bibliographic references may be examined. Nutr Hosp 2008; 23:85-8.

28. Miralles J, Ramos JM, Ballester R, Belinchón I, Sevilla A, Marangón M. Estudio bibliométrico de la revista Actas Dermo-Sifiliográficas (1984-2003) II. Análisis de las referencias bibliográficas. Actas Dermosifiliogr 2005; 96:563-71.

29. Villar Álvarez F, Estrada Lorenzo JM, Pérez Andrés C, Rebollo Rodríguez MJ. Estudio bibliométrico de los artículos originales de la revista española de salud pública (1991-2000). Parte tercera: análisis de las referencias biblográficas. Rev Esp Salud Pública 2007; 81:247-59.
30. Casterá VT, Sanz-Valero J, Juan-Quilis V, WandenBerghe C, Culebras JM, García de Lorenzo y Mateos A. Estudio bibliométrico de la revista Nutrición Hospitalaria en el periodo 2001 a 2005: parte II, análisis de consumo; las referencias bibliográficas. Nutr Hosp 2008; 23:541-6.

31. Lobanco CM, Vedovato GM, Cano C, Bastos DHM. Reliability of food labels from products marketed in the city of Sao Paulo, Southeastern Brazil. Rev Saúde Pública 2009; 43:499-505.

32. Harnack LJ, French SA. Effect of point-of-purchase calorie labeling on restaurant and cafeteria food choices: a review of the literature. Int J Behav Nutr Phys Act 2008; 5:51.

33. Kolodinsky J, Grren J, Michahelles M, HarveyBerino JR. The use of nutritional labels: students in a food-court by college setting. J Am Coll Health 2008; 86:297-301.

34. Wansink B, Chandon P. Can "low-fat" nutrition labels lead to obesity? J Mark Res 2006; 43:605-17.

35. Jain A, Sherman SN, Chamberlin LA, Carter Y, Powers SW, Whitaker RC. Why don't low-income mothers worry about their preschoolers being overweight? Pediatrics 2001; 107:1138-46.

36. Dumanovsky T, Nonas CA, Huang CY, Silver LD, Bassett MT. What people buy from fast-food restaurants: caloric content and menu item selection, New York City 2007. Obesity 2009; 17:1369-74.

37. Rydell SA, Harnack LJ, Oakes JM, Story M, Jeffery RW, French SA. Why eat at fast-food restaurants: reported reasons among frequent consumers. J Am Diet Assoc 2008; 108:2066-70.

38. O’Dougherty M, Harnack LI, French SA, Story M, Oakes JM, Jeffery RW. Nutrition labeling and value size pricing at fast-food restaurants: a consumer perspective. Am J Health Promot 2006; 20:247-50.

39. Adams C. Reframing the obesity debate: McDonald's role may surprise you. J Law Med Ethics 2007; 35:154-7.

40. Away-from-home foods: final report released by the keystone forum. Health Care Food Nutr Focus $2006 ; 23: 10-2$.

41. Elliot C. Assessing "fun foods": nutritional content and analysis of supermarket foods targeted at children. Obes Rev 2008; 9:368-77.

42. Hawkes C. Agro-food industry growth and obesity in China: what role for regulating food advertising and promotion and nutrition labelling? Obes Rev 2008; 9 Suppl 1:151s-61s.

43. Hignett R. Labelling to get noticed. Community Pract 2007; 80:12-3.

44. Krukonski RA, Harvey-Berino JR. Need for an effectiveness of menu labeling: authors' response. J Am Diet Assoc 2007; 107:33-4.

45. Borgmeier I, Westenhoefer J. Impact of different food label formats on healthiness evaluation and food choice of consumers: a randomized-controlled study. BMC Public Health 2009; 9:184.

Recibido el 30/Ago/2010

Versión final presentada el 28/Abr/2011

Aprobado el 01/Jun/2011 